\title{
Quantification of radiative attenuation provided by fire hose nozzles
}

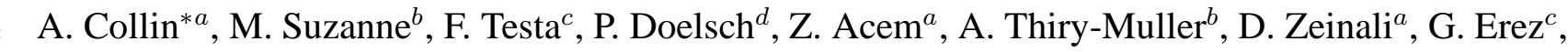

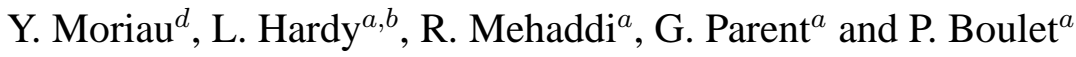 \\ a: Université de Lorraine, CNRS, LEMTA, F-54000 Nancy, France \\ b: LCPP, Préfecture de Police de Paris, F-75015 Paris, France \\ $c$ : Brigade de Sapeurs-Pompiers de Paris, F-75017 Paris, France \\ d: Service Départemental d'Incendie et de Secours de Meurthe et Moselle, F-54270 Essey-lès-Nancy, France
}

8 KEY WORDS: radiative attenuation, nozzle, fire fighting, thermal protection

\begin{abstract}
9 The aim of this study was to investigate the radiative protection provided by various fire hose nozzles used by several Fire

10 Rescue Services in France and to propose an experimental set-up to quantify it. This study combined the use of radiative 11 sources (a radiant panel or a fire inside a standard shipping container) and metrological devices (radiative heat sensors,

IR camera and spectrometer) to estimate the radiative attenuation of water sprays used to protect the firefighters against thermal effects which occur during a fire. For all the fire hose nozzles tested in this work, the maximal effective attenuation reaches $75 \%$. For most of them, an increase of the flow rate improves the radiative attenuation. However, this study shows that a similar attenuation can be reached for different flow rates, suggesting that the droplet size diameter and the droplet volume fraction also play a significant role in the efficiency of the provided spray.
\end{abstract}

${ }^{*}$ Correspondence to: anthony.collin@univ-lorraine.fr 


\section{INTRODUCTION}

1 The fire hose nozzles are one of the most utilised pieces of a fire fighting equipment. They are used to spray water, in front

of, in the vicinity of or onto a fire. Särdqvist [1] summarized that water can be used to attack fire in five different ways: to cool hot smokes, to cool/extinguish flames, to cool fuel surfaces to stop the pyrolysis process, to keep the fuel surfaces not yet involved in fire cool and to vaporise on hot surfaces to inert the fire by water steam. For firefighting operations, these nozzles can be used differently depending on their flow patterns:

- Straight stream: in this configuration, the firefighters can work far away from solid fuel fires and can use the kinetic energy to eliminate embers and to reach fire pockets.

- Variable fog patterns: the fine droplets generated are used to rapidly cool the environment (smoke and hot gases) through water vaporisation or to dilute the smoke in a room in order to avoid any ignition hazards.

- Protective shield: the spray generated has a wide angle and is composed of fine water droplets. This spray should guarantee a protection of firefighters against thermal radiation, especially against flashover occurrences for instance.

In France, three standards $[?, ?, ?, ?, ?, ?, ?, ?, 2,3]$

Despite the fact that these nozzles are common tools for firefighters, few scientific studies have been performed to assess the efficiency of these extinction devices. To the best of our knowledge, the most recent studies on fire hose nozzle mainly concern the evaluation of the "backward reaction", as treated by Vera et al. [5,6], Chin et al. [7] or Sun et al. [8]. However, no particular literature is devoted to the thermal efficiency of hose nozzles for firefighting operations.

The present study focuses on the nozzle efficiency to generate a water spray which protects the firefighters against thermal radiation emitted by a hot source, such as flames or smoke.

The capability of a water spray to attenuate radiation coming from a fire has been highlighted in a pioneering work by Dembélé et al. [9], followed by others such as the experimental and numerical results presented by Collin et al. [10-12]. These studies illustrated that the radiative attenuation by the spray is obtained by a combination of absorption and scattering phenomena by water droplets. The efficiency of a spray to attenuate thermal radiation depends on several factors: 
- the droplet size distribution ;

- the droplet volume fraction ;

- the water spray geometry ;

- the spectral range of the radiative source.

Absorption and scattering are both involved in the attenuation effect. Their respective contribution depends on the droplet size and concentration. In the case of large droplets (larger than $100 \mu \mathrm{m}$ diameter), scattering is strongly anisotropic, with a sharp forward-oriented phase function, meaning a weaker efficiency for radiation shielding [10]. This is why absorption is considered as the main contributor of the effective radiation attenuation. The results presented in these studies are limited to weak water flow rates (several litres per minute). For fire hose nozzle applications, the flow rates can vary from several dozens up to several hundred litres per minute.

To provide some estimations of the waterspray thermal protection, Tseng and Viskanta [13] proposed empirical equations to predict spectral absorptance and transmittance of water droplet monodispersions and polydispersions. Their works considered different droplet diameters from $25 \mu \mathrm{m}$ to $250 \mu \mathrm{m}$ and for droplet loading levels $N \cdot L$ ( $N$ the total number of particles in unit volume and $L$ the waterspray width) from $10^{4}$ to $10^{11}$ droplets $/ \mathrm{m}^{2}$. These used ranges in term of droplet diameters seem to be out of scope for the present application (fire hose nozzles).

Mehaddi et al. [14] derived a simplified model to predict the total attenuation of a waterspray knowing its mean features. These relations are function of the droplet volume fraction, their size distribution and the spray dimensions. However, for fire hose nozzle applications, these parameters are hardly available and are not experimentally measurable, due to the high flow rates used (from 250 up to 1250 1/min) and the large dimensions of the spray (several square meters).

As mentioned before, the efficiency of a waterspray to block radiations from a hot source depends on the volume fraction of water droplets, their size distribution and the spray dimensions. To determine these parameters, there are several kinds of laser diagnostics, such as PDA technique (extension of laser Doppler anemometry) or laser diffractometer. However, for fire hose nozzle applications, due to the high flow rates used and the large dimensions of the spray (several square meters), these experimental devices can not be set up easily: for PDA technique, the optical plane (containing the emitter and receptor sensor) should be orthogonal to the droplet trajectories (which are horizontal); or for laser diffraction method, 
the measurement is performed on an optical path (providing a mean data, not local) which is smaller than the spray pattern dimensions. That is why, the droplet volume fraction and their size distribution are hardly available and measurable with experimental means. It is therefore preferable to measure directly the radiative attenuation instead, without worrying about the drop distribution.

The aim of the present study is thus to quantify experimentally the ability of a fire hose nozzle to generate a water spray which efficiently protects the firefighters against radiation emitted by hot sources. To the best of our knowledge, a study with full-size sprays for fire hose nozzles has never been carried out. The efficiency of the radiative shield is achieved by quantifying the radiative attenuation of each water spray with dedicated measurement sensors, including a FTIR spectrometer and an IR camera. A second objective is to propose an experimental set-up allowing the estimation of the radiative attenuations in a simple way (in considering only the metrology aspect, not for the experimental setup).

The paper is organized as follows: Section 1 briefly recalls the main concepts based on the Mie theory, to explain how the water droplets attenuate radiations and what are the mean features; Section 2 presents the selected fire hose nozzles and their characteristics; Section 3 describes the experimental set-up and the attenuation calculation made in this study to quantify the capability of a water spray to block radiation; Section 4 presents some results on spectral directional attenuations obtained with a small size radiative source; Section 5 is dedicated to the estimation of the extended attenuation provided by a water spray on a real fire; Section 6 draws our conclusions and some perspectives for future works.

\section{THEORETICAL ASPECTS ON RADIATIVE ATTENUATION BY WATER DROPLETS}

In applying theoretical aspects of the Mie theory [15], in a recent works [14], we derived a simplified model to predict the total attenuation of a waterspray knowing its mean features. As the radiative attenuation provided by these water sprays is mainly due to absorption phenomena, the total attenuation (noted here $A$ ) can be estimated knowing the width of the spray (denoted here by $L$ ) and using Beer's law [16],

$$
A=1-\exp (-\beta L)
$$

where $\beta$ is the effective extinction coefficient representing the radiative attenuation of the waterspray. These parameters take into account the combined effects of the droplet diameter and the droplet volume fraction. $\beta$ can be considered as a 

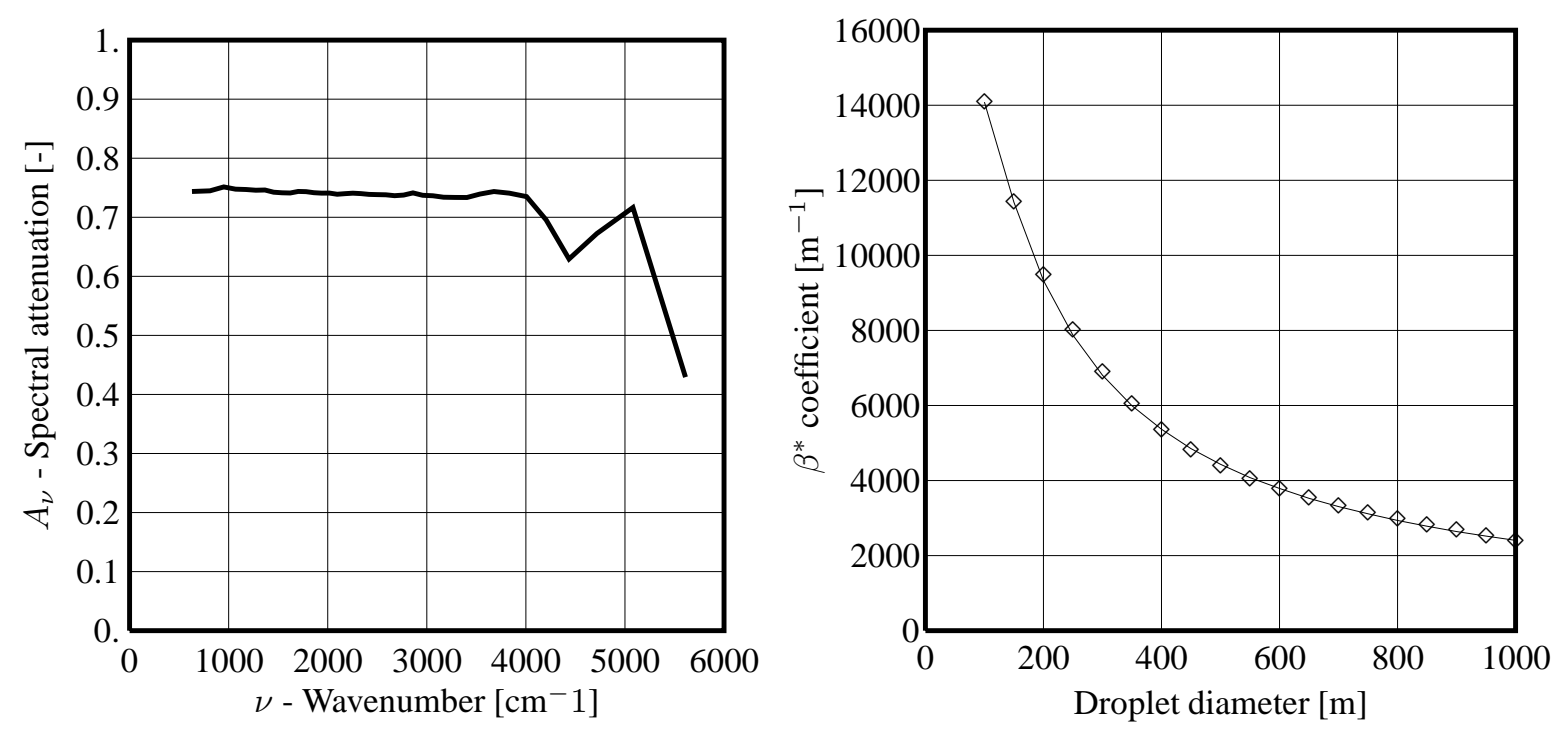

(a) Spectral radiative attenuation of a fictitious waterspray (mean (b) $\beta^{*}$ representation function of droplet diameter: the results of Mie diameter: $500 \mu \mathrm{m})$ theory computation are in symbols and an attempt to provide an approximation by a least square method in solid line.

Figure 1 Examples of results on the radiative transfer, for a waterspray width of $4 \mathrm{~m}$ and a droplet volume fraction of $5 \cdot 10^{-5} \mathrm{~m}^{3} / \mathrm{m}^{3}$

spectral feature, and in this way, $A$ becomes a spectral attenuation in Eq.(1).

2 Based on the combination of the Mie theory [15] and a Monte Carlo Method to treat numerically the radiative heat 3 transfer [14], the spectral attenuation of a fictitious waterspray (whose main features are mean diameter, 500 $\mu$ m, spray 4 width, $4 \mathrm{~m}$, and droplet volume fraction, $5 \cdot 10^{-5} \mathrm{~m}^{3} / \mathrm{m}^{3}$ ) can be estimated and presented in Figure 1(a). Obviously, this 5 spectral attenuation, and then the extinction coefficient, weakly vary despite the wide range of wavenumber. The spectral 6 extinction coefficient, $\beta_{\nu}$, defined by the Mie theory is given by,

$$
\beta_{\nu}=\pi \frac{d^{2}}{4} N Q_{\nu \mathrm{ext}}(d)
$$

8 where, $d$ is the droplet diameter (or mean droplet diameter), $N$ the total number of particles in unit volume and $Q_{\nu \text { ext }}$ the 9 efficiency coefficients for extinction provided by Mie theory [15], for instance. For the sake of clarity, these expressions are considered in the form of a mono-disperse spray with a uniform (mean) droplet size distribution. Other expressions can 11 be found when a specific droplet distribution is considered [10]. As $N=6 \frac{f_{v}}{\pi d^{3}}$, the spectral coefficient can be estimated 
from the droplet volume fraction by,

$$
\beta_{\nu}=f_{v} \beta_{\nu}^{*}
$$

3 where $\beta_{\lambda}^{*}$ depends only on the droplet diameter and is expressed as,

$$
\beta_{\nu}^{*}=\frac{3}{2 d} Q_{\nu \mathrm{ext}}(d)
$$

5 As it has been previously shown that the spectral attenuation weakly varies on the IR domain (Cf. Figure 1(a)), the total

6 attenuation can be estimated by,

$$
A=1-\exp \left(-\beta^{*} f_{v} L\right)
$$

8 For fire hose nozzle applications, the droplet volume fraction, noted here $f_{v}$ depends directly on the water flow rate used 9 and the feeding pressure at the fire hose nozzle.

10 For a nominal configuration with a droplet volume fraction set to $5 \cdot 10^{-5} \mathrm{~m}^{3}$ of water $/ \mathrm{m}^{3}$ of air and a spray width to 4 $11 \mathrm{~m}$, different situations were considered by varying the droplet diameter from $100 \mu \mathrm{m}$ to $1000 \mu \mathrm{m}$. Figure 1(b) represents,

\section{SELECTED FIRE HOSE NOZZLES}

Four fire hose nozzles were tested in this work. They were selected from several Fire Rescue Services in France. The manufacturer names will not be given in this paper for confidentiality reasons: the nozzles are referenced from Nozzle \#1 to Nozzle \#4.

Each nozzle was connected to several lengths of $45 \mathrm{~mm}$ hoses. The fire engine pump guaranteed that the operating pressure at the fire hose nozzle was set at 6 bars allowing to compensate the pressure losses inside the fire hoses and connections. This pressure was measured by a manometer placed before the fire hose nozzle. 


\begin{tabular}{|c|c|c|c|c|c|c|c|c|c|}
\hline \multirow{2}{*}{$\begin{array}{l}\text { Flow rate } \\
\text { selected }\end{array}$} & \multicolumn{3}{|c|}{ Nozzle \#1 } & \multicolumn{2}{|c|}{ Nozzle \#2 } & \multicolumn{3}{|c|}{ Nozzle \#3 } & \multirow{2}{*}{$\frac{\text { Nozzle \#4 }}{\dot{Q}_{\text {mea }}}$} \\
\hline & $\dot{Q}_{\min }$ & $\dot{Q}_{\text {int }}$ & $\dot{Q}_{\max }$ & $\dot{Q}_{\min }$ & $\dot{Q}_{\max }$ & $\dot{Q}_{\min }$ & $\dot{Q}_{\text {int }}$ & $\dot{Q}_{\max }$ & \\
\hline $\begin{array}{l}\text { Measured } \\
{[l / \min ]}\end{array}$ & [200-240] & [295-400] & [570-620] & [330-380] & [500-660] & [85-110] & [235-250] & [350-390] & 100 \\
\hline
\end{tabular}

Table 1 Values of flow rates for each fire hose nozzle used in this study

1 For each fire hose nozzle, one or several flow rates were tested in accordance with the manufacturer recommendations to

2 generate a radiative protection shield: minimal flow rate (denoted by $\dot{Q}_{\text {min }}$ ), maximal flow rate (denoted by $\left.\dot{Q}_{\text {max }}\right)$ and,

3 if available, an intermediate case (denoted by $\dot{Q}_{\text {int }}$ ). For Nozzle \#4, one unique flow rate has been selected called $\dot{Q}_{\text {mea. }}$.

4 The selected flow rates were identified using the indications on the nozzle. Table 1 summarises the values of the effective

5 flow rate measured during the experiment. In this study, the considered flow rates cover a wide range, from $851 / \mathrm{min}$ to

$6 \quad 6601 / \mathrm{min} \pm 21 / \mathrm{min}$. Note that a minimum of $5001 / \mathrm{min}$ at 6 bars was required for all nozzles used by rescue services for

7 fighting indoor fires by French regulations until 2018.

\section{EXPERIMENTAL SET-UP}

8 The experimental set-up is defined as follows: the fire hose nozzle is located between a radiant source (a radiant panel

9 or a real fire) and the measuring device, namely a spectrometer, an IR camera, or a heat gauge sensor. Figure 2 gives an

10 overview of the experimental set-up.

11 During each test, the spray was activated for $30 \mathrm{~s}$. The experiments for each nozzle and flow rate were performed three 12 times to verify the repeatability of the measurements. The final results were reported in terms of average values and their 13 standard mean deviations.

14 The efficiency of the protective shield is defined by its radiative attenuation computed such as:

15

$$
A=1-\frac{S_{\text {with spray }}-S_{\text {ambient }}}{S_{\text {without spray }}-S_{\text {ambient }}}
$$
where $A$ is the radiative attenuation ranging from 0 (i.e., no attenuation) to 1 (i.e., $100 \%$ attenuation) and $S$ is the radiative 17 signal measured at the firefighter position, behind the fire hose nozzle. $S$ represents a radiative heat flux, which is a voltage 
1 recorded by the radiative sensor, a pixel value for the infrared camera or a dimensionless level for the spectrometer. $S_{\text {ambient }}$

2 is the radiative reference signal without a spray and without a radiative source, $S_{\text {without spray }}$ the one without a spray but

3

4

5

6

7

8 experimental device has been calibrated with a blackbody emitter.

\section{SPECTRAL AND DIRECTIONAL ATTENUATION ESTIMATION}

${ }_{20} \quad I_{\nu}=\frac{S}{S^{0}} I_{\nu}^{0}\left(T_{b b}\right)$

with a radiative source and $S_{\text {with spray }}$ the one with a spray and a radiative source. Depending on which measurement device is used, these features can be considered as spectral or total quantities to estimate spectral attenuation $\left(A_{\nu}\right)$ or total attenuation $(A)$ respectively. Note that $S_{\text {ambient }}$ was measured before each experiment in ambient conditions. The radiative attenuation was calculated when the measured radiative signal (heat flux for instance) was constant over time. This choice avoids any transient state which occurs just after the activation of the spray. Before this experimental campaign, each

In this section, we present the experiments made to characterize the spectral attenuation obtained with sprays generated by several fire hose nozzles. The measurements were carried out with a Fourier Transform InfraRed (FTIR) spectrometer (Matrix from Bruker) combined with a multi-spectral camera (ORION SC7000 from FLIR) to provide spectral radiative attenuations. The spectrometer detectors allowed measurements in the range between 600 and $5000 \mathrm{~cm}^{-1}$, and the spectrometer resolution was set to $4 \mathrm{~cm}^{-1}$.

Concerning the results provided by the spectrometer, 10 instantaneous values were averaged to improve the signal to noise ratio. The spectrometer solid angle of detection is $8.5 \cdot 10^{-6} \mathrm{sr}$, which corresponds to an angular acceptance of $0.35^{\circ}$. Considering the distance between the spectrometer and the radiative source, the detection area is negligible compared to that of the radiant panel. The radiative attenuation was measured along the spray centre-line. The signal provided by the spectrometer (here denoted by $S$ ) is compared with that provided by a black body (denoted by $S^{0}$ ) at $1173 \mathrm{~K}$ (named $T_{b b}$ ) under the same geometrical configuration. The spectral intensity (denoted by $I_{\nu}$ ) is then computed as follows:

The IR camera was equipped with a band-pass filter centred at the wavenumber of $2564 \mathrm{~cm}^{-1}$ to avoid any emission / absorption band of participating gases, such as $\mathrm{CO}_{2}, \mathrm{CO}$ or $\mathrm{H}_{2} \mathrm{O}$. For the analysis, the detection area of the infrared camera was set to match with the target viewed by the spectrometer. For each pixel, data provided by the infrared camera is a pixel 


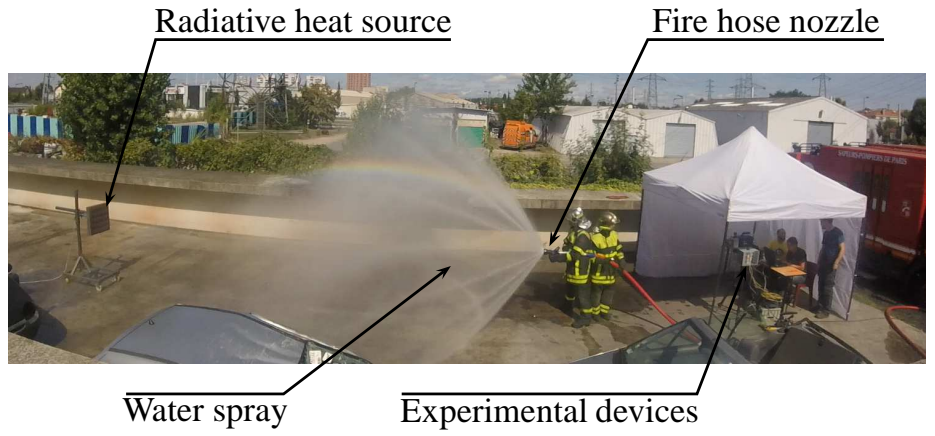

(a) Lateral view

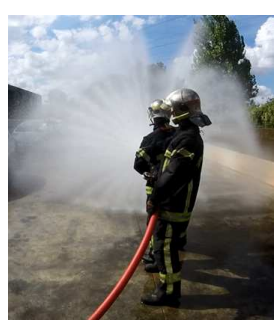

(b) Rear view

Figure 2 Experimental set-up in tests with a radiant panel

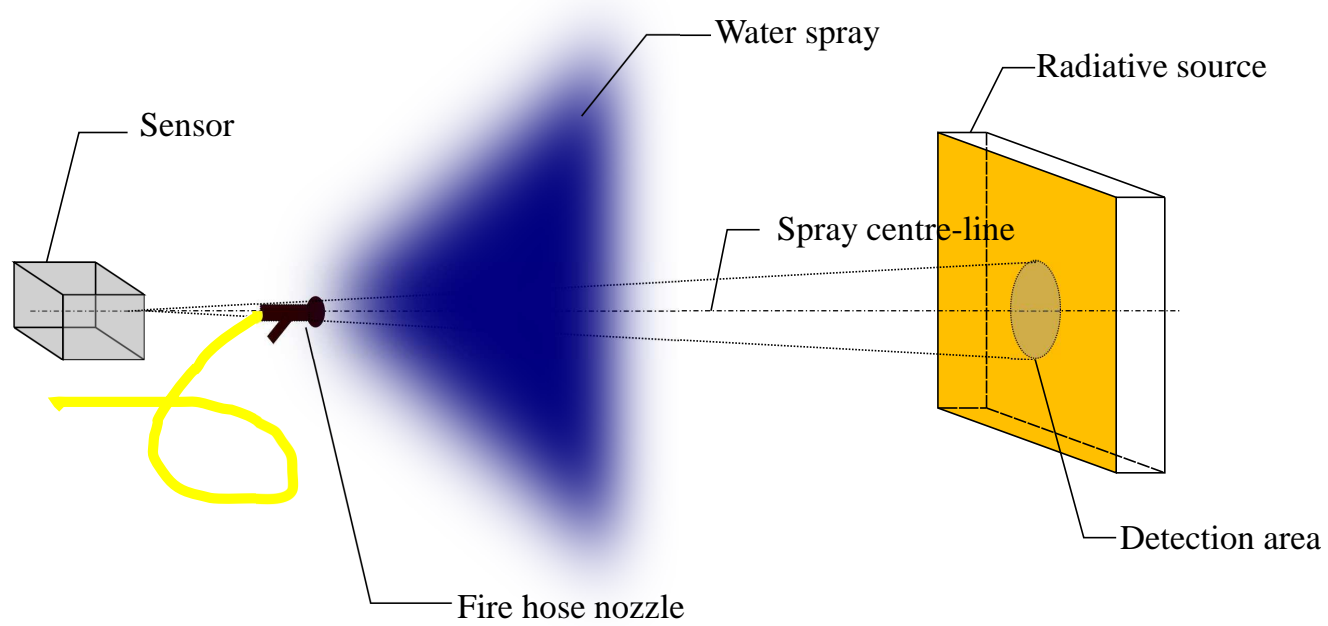

Figure 3 Schematic representation of the experimental set-up

1 value which is proportional to the sum of a background value and of the received radiation. Because the signal $S_{\text {ambient }}$ 2 is subtracted in Eq.(6) the background value cancels out and the ratio is therefore equal to the ratio of the flux received 3 with and without the spray. The pixel values belonging to the spectrometer detection area are summed to correspond to 4 the measurement made by the spectrometer. In order to reduce noise and effects of fluctuations (coming mainly from the 5 spray which is not perfectly stable), the signal was averaged for $10 \mathrm{~s}$.

6 The radiative source is a radiant panel fed with butane. Its dimensions are $50 \times 50 \mathrm{~cm}^{2}$, and it is located at $6 \mathrm{~m}$ from the 
7 fire hose nozzle and $10 \mathrm{~m}$ from the measurement sensors. An overview picture is given in Figure 2(a) where the radiant 8 panel is visible on the left-hand side of the image. Figure 2(b) is a rear view of the spray generated by the nozzle. Figure 93 gives a schematic representation of the experimental setup. In this Figure, the sensor can be the radiative sensor, the 10 spectrometer or the IR camera. The Heat Release Rate (HRR) provided by the radiant panel is about $50 \mathrm{~kW}$ according 11 to the manufacturer. Using a radiative heat flux sensor, the radiative heat flux emitted by the radiant panel was measured 12 at $70 \mathrm{~kW} / \mathrm{m}^{2}$, that corresponds to a $17.5 \mathrm{~kW}$ radiation power emitted by the panel ( $35 \%$ of total HRR). The other part, $1332.5 \mathrm{~kW}$, concerns the convective heat transfer and this is constant over time as the measured radiative heat fluxes imply. 14 The distance between the radiative source and the fire hose nozzle is high enough to prevent any water deposition on the 15 hot source. Results are provided as a function of the wavenumber which is the inverse of the wavelength. The emission / 16 absorption bands around $1500 \mathrm{~cm}^{-1}$ and $3750 \mathrm{~cm}^{-1}$ are due to the water vapour, and the variations centred at $2300 \mathrm{~cm}^{-1}$ 17 are the combined effects of $\mathrm{CO}_{2}$ (emission by the source and absorption by the atmospheric gases along the line of sight). 1 In Figure 4(a), the spectral band-pass filter of the IR camera (centred at the wavenumber of $2564 \mathrm{~cm}^{-1}$ ) is highlighted 2 with a grey line to indicate that no participating gas influences the measurements performed with the infrared camera at 3 this wavenumber. Note that the radiant panel is solely used as a thermal radiation emitter, and thus the convective heat 4 release rate it provides does not matter.

5 Figure 4(a) gives an example of the spectral intensity obtained with the spectrometer when using Nozzle \#1 at different flow rates. All of the proposed spectra follow the same trend: a global profile with some additional emission / absorption bands of participating gases. For Nozzle \#1, these spectral intensities indicate that the radiative level decreases with an increase in the flow rate.

$9 \quad$ Figure 4(b) shows the spectral attenuation estimated with Eq. (6) based on data provided by the spectrometer for Nozzle 10 \#1. It can be seen that the spectral attenuation varies only slightly as a function of the wavenumber, weakly decreasing 11 as the wavenumber increases. That indicates that the droplets diameter is large compared to the wavelength. Indeed for droplets diameter similar to the wavelength, the extinction efficiency becomes more wavelength dependent as predicted theoretically by the Mie theory [15] and observed experimentally [17]. This also applies for the other fire hose nozzles 14 (other spectral attenuations are not presented here). This indicates that in the IR domain, the water spray generated by the 


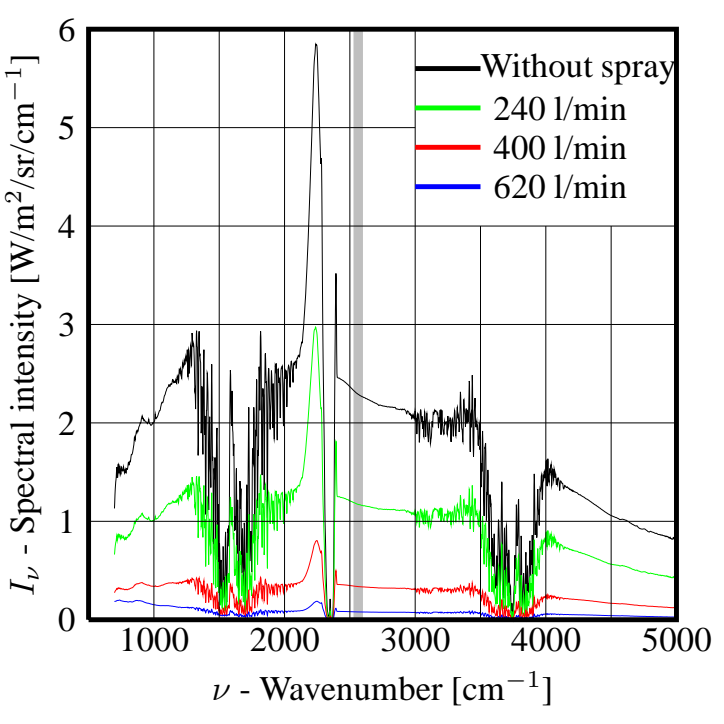

(a) Spectral intensities emitted by the radiant panel through the waterspray and measured by spectrometer for several flow rates for Nozzle \#1

Figure 4 Examples of measurements performed with the spectrometer for Nozzle \#1. The grey band corresponds to the IR domain covered by the band-pass filter centred at the wavenumber of $2564 \mathrm{~cm}^{-1}$

nozzles almost behaves as a grey medium. For the repeatability test, the highest discrepancies are observed for Nozzle \#1 at $2401 / \mathrm{min}$, where the relative variations are approximately $20 \%$. Increasing the water flow rate results in a better radiative attenuation, varying from a mean value of $50 \%$ for 240 1/min to $95 \%$ for 620 1/min. Similar results were observed in [18]

4 for the spectral directional attenuation estimations performed on a water mist at lower flow rates. Data presented in Figure

54 (b) show that a radiation attenuation close to $100 \%$ can be achieved with a fire hose nozzle, provided that the flow rate is 6 high enough or that the spray characteristics are suitable.

7 It is useful to define an average attenuation $(\bar{A})$ to compare the fire hose nozzle performance on the basis of a single value.

8 Such average values must be computed by weighing the integral of the spectral attenuation against the spectral distribution

9 of the radiative source. For a black body, this yields the so-called Planck's mean [16] as follows: 


\begin{tabular}{|c|c|c|c|c|c|c|c|c|c|}
\hline \multirow{2}{*}{$\begin{array}{l}\text { Nozzle } \\
\text { Measured flow rate }[1 / \mathrm{min}]\end{array}$} & \multicolumn{3}{|c|}{$\# 1$} & \multicolumn{2}{|c|}{$\# 2$} & \multicolumn{3}{|c|}{ \#3 } & \multirow{2}{*}{$\frac{\# 4}{100}$} \\
\hline & 240 & 400 & 620 & 380 & 660 & 85 & 235 & 350 & \\
\hline $\bar{A}[\%]$ (source temperature $T=1000 \mathrm{~K}$ ) & 50 & 86 & 96 & 99 & 99 & 90 & 98 & 98 & 99 \\
\hline $\begin{array}{l}\text { Relative variation of } \bar{A}[\%] \text { for a } \\
\text { source temperature } T=[800-1200 \mathrm{~K}]\end{array}$ & 3.0 & 0.8 & 0.3 & 0.1 & 0.1 & 0.8 & 0.1 & 0.1 & 0.2 \\
\hline
\end{tabular}

Table 2 Comparison of the mean directional radiative attenuation $(\bar{A})$ for a radiative source temperature set at $1000 \mathrm{~K}$ and its sensitivity according to the source temperature.

$$
\bar{A}=\frac{\int_{\Delta \nu} A_{\nu} I_{\nu}^{0}(T) \mathrm{d} \nu}{\int_{\Delta \nu} I_{\nu}^{0}(T) \mathrm{d} \nu}
$$

where $\bar{A}$ is the average attenuation, $I_{\nu}^{0}(T)$ is the Planck's black body intensity [16] estimated at a given wavenumber $\nu$ and at a temperature $T$, and $\Delta \nu$ is the integration domain defined between $600 \mathrm{~cm}^{-1}$ and $5000 \mathrm{~cm}^{-1}$. Based on Eq. (7), mean values of attenuation were estimated for a radiative source temperature set at $1000 \mathrm{~K}$. The results are summarised in Table 2.

Based on the results presented in Table 2, Nozzle \#2 and Nozzle \#4 can generate very efficient radiative shields (close to $100 \%$ attenuation) despite differences in their water flow rates, from 100 1/min for Nozzle \#4 up to 660 1/min for Nozzle \#2. The radiative efficiency of these two fire hose nozzles can be explained visually by smaller water droplets they produce compared to Nozzle \#1 and \#3 (no particle size analysis is available in this work to quantify the droplet size distributions).

The average values of radiative attenuation summarised in Table 2 do not indicate a significant dependence on the radiative source temperature. Indeed, Table 2 gives for each fire hose nozzle and each flow rate, the relative variations of the radiative attenuation when considering a source temperature varying between $800 \mathrm{~K}$ and $1200 \mathrm{~K}$. The relative discrepancy in the level of attenuation is lower than $3 \%$, confirming that the spectral distribution of the radiative source does not impact the efficiency of the water sprays (due to the grey behavior of radiative attenuation previously discussed).

In the following paragraphs, the measurements performed with the spectrometer are compared with the ones provided by 


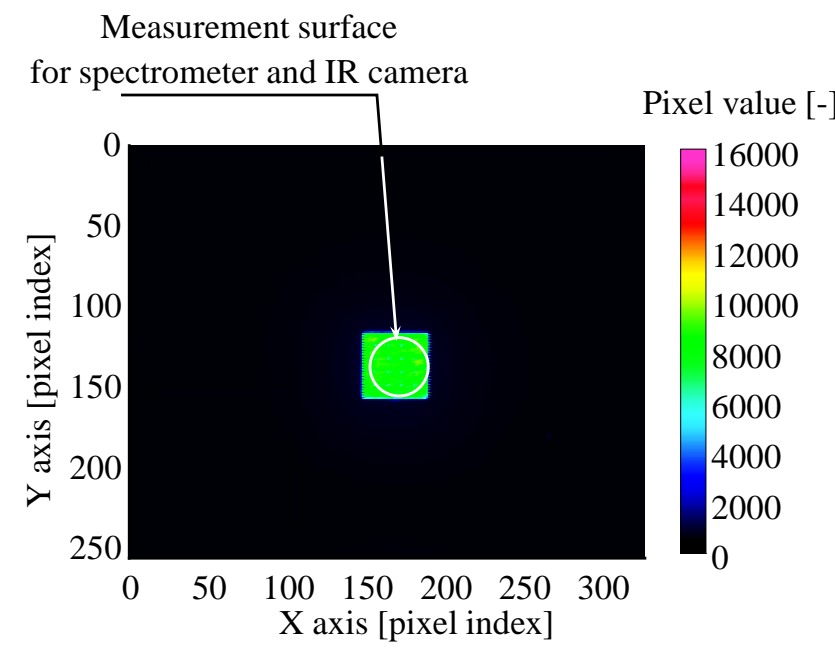

(a) Screenshot from IR camera viewing the radiant panel. The white circle corresponds to the measurement area used by the spectrometer

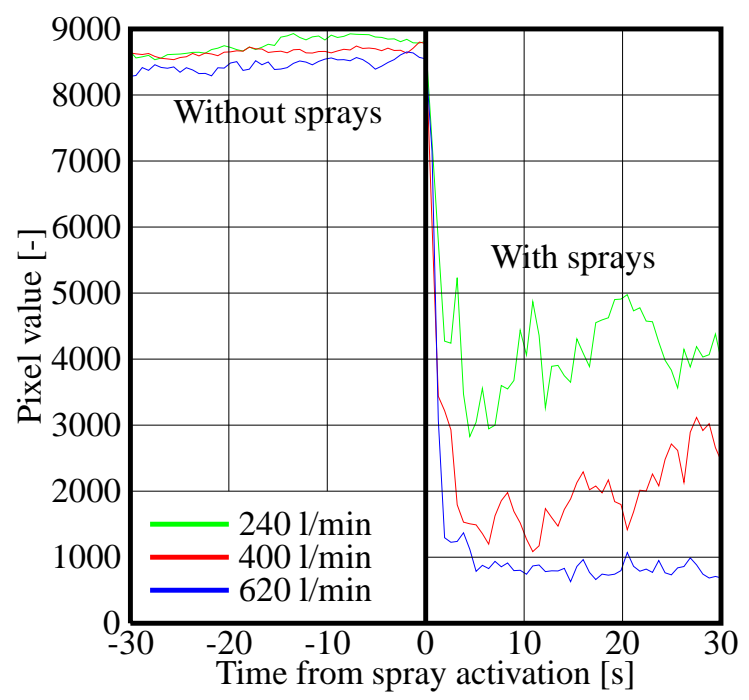

(b) Evolution of mean pixel values on the measurement surface equivalent to that for the spectrometer during one water spray activation.

Figure 5 Examples of raw data from the IR camera for Nozzle \#1. $X$ and $Y$ axis denote the horizontal and vertical directions respectively

the IR camera. Figure 5(a) gives an illustration of the radiant panel as viewed by the IR camera. The white circle in Figure 5(a) represents the measurement area considered by the spectrometer. To estimate the radiative attenuation, the analysis of the results given by the IR camera focuses on the same measuring area. The signal received by the IR camera, in pixel value, is averaged over this area.

Figure 5(b) plots the shifted results for the time axis $(0 \mathrm{~s}$ corresponds to the water spray activation) measured by the IR camera for the three flow rates used with Nozzle \#1. Before the spray activation, the signals are relatively constant, showing the good stability of the radiative source. After the radiative shield activation, the results present the same trend as the ones observed with the spectrometer: the pixel value decreases when the flow rate increases. When the water spray is active, the IR camera levels may present some variations in time due to the movements of the operator holding the fire hose nozzle and the variations generated by the fire engine pump to maintain the water flow rate. The consequence is that the shape of the spray, and therefore the radiative shielding, varies over time (observed with pictures taken with a visible camera, not presented here) thus modifying the pixel value obtained with the IR camera. 


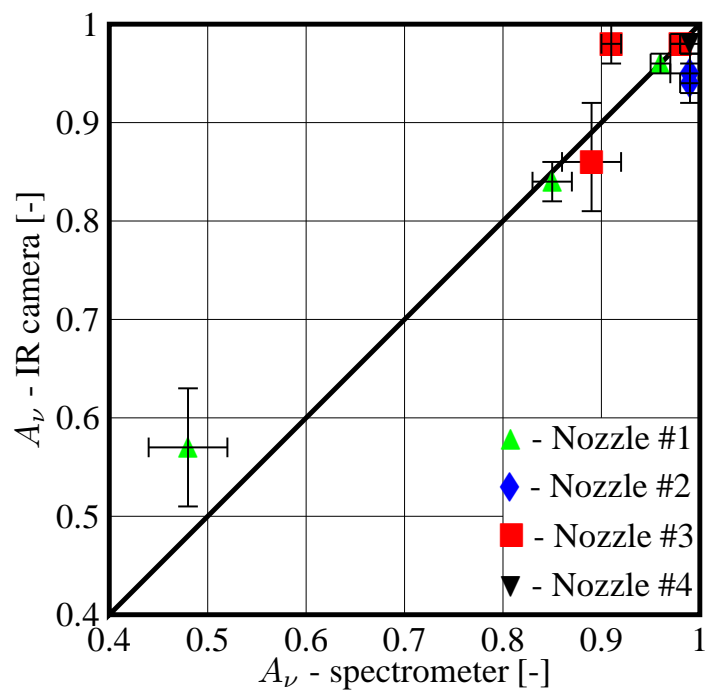

Figure 6 Comparison of levels of spectral radiative attenuation $\left(A_{\nu}\right)$ determined based on spectrometer and IR camera measurements at the wavenumber of $2564 \mathrm{~cm}^{-1}$

In order to compare the results obtained with the spectrometer and the camera, the spectral attenuations provided by both experimental tools at the wavenumber of $2564 \mathrm{~cm}^{-1}$ are plotted in Figure 6. It can be observed that the spectral attenuation predictions are similar, with a mean discrepancy of $4 \%$ and a maximal one of $15 \%$. This data confirms that the results 2 provided by the spectrometer and the IR camera are in a good agreement in terms of the quantified efficiency of water 3 sprays for shielding against thermal radiation.

4 As depicted in Figure 2(b), a large amount of droplets appears to be concentrated at the spray centre which is the 5 area currently used to measure the attenuations. In these conditions, the directional radiative attenuation is certainly 6 overestimated and the quantified levels might overestimate the actual protection provided by the water sprays. This is why 7 the second part of this work focuses on estimating the extended radiative attenuation of water sprays generated by fire 8 hose nozzles with an extended radiative source, such as a real fire.

\section{EXTENDED RADIATIVE ATTENUATION QUANTIFICATION}

9 The radiant panel was replaced by a "real" fire inside a standard shipping container ("20 feet dry container") with 10 dimensions $6.10 \times 2.43 \times 2.59 \mathrm{~m}^{3}$. The fire in the container was created with 16 wooden pallets stacked in two 


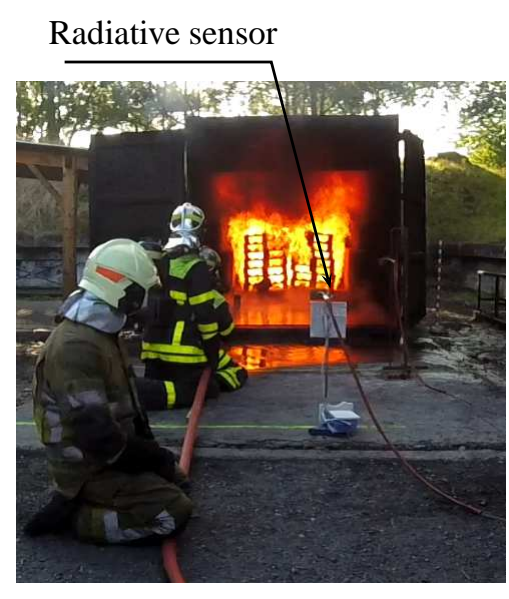

(a) Rear view of the experimental set-up without water

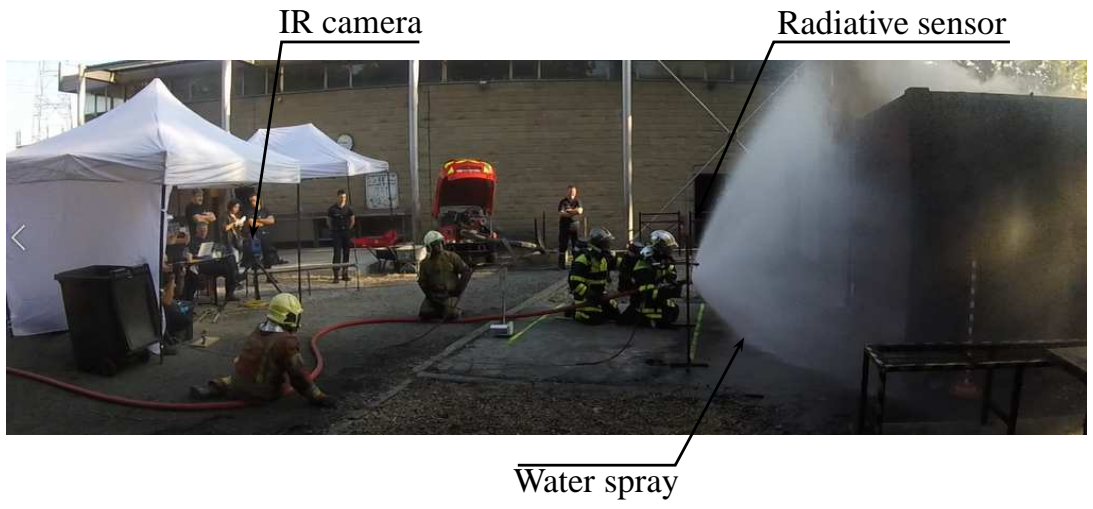

(b) Lateral view of the experimental set-up during a water spray activation spray activation

Figure 7 Experimental configuration for the extended radiative attenuation estimation

separate columns as shown in Figure 7(a). Each pallet measured $120 \times 80 \times 17 \mathrm{~cm}^{3}$ and the total combustible mass was approximately $375 \mathrm{~kg}$. Ignition was performed at the ground level, in the bottom right corner of the container. This load produced a $1200 \mathrm{~s}$ long flaming fire which was fully developed $700 \mathrm{~s}$ after the ignition. Figure 7 gives a global view of the experimental set-up. The experiments were repeated three times to verify the reliability of the results.

In this configuration, the firefighters were located at a distance of $2 \mathrm{~m}$ from the front of the container. A total radiative heat flux meter (from CAPTEC) was placed next to the firefighters, $1 \mathrm{~m}$ from the ground (next to the fire hose nozzle). The IR camera was set $8 \mathrm{~m}$ away from the container. The relative position of each experimental device is shown in Figure 7(b).

For the tests concerned with the extended radiative attenuation (as a hemispherical feature) estimation, the water supply system was different from that in the previously discussed tests. This is why, for the same selected flow rate, the "real" flow rate can differ from the ones measured in the preceding analysis with the radiant panel.

An image obtained with the IR camera is given in Figure 8(a). In this figure, the fire is not homogeneous in terms of radiative contributions and the hot wooden pallets can be distinguished from the container. In Figure 8(a), a white circle shows the equivalent area which corresponds to the spectrometer's solid angle of detection. The directional radiative 


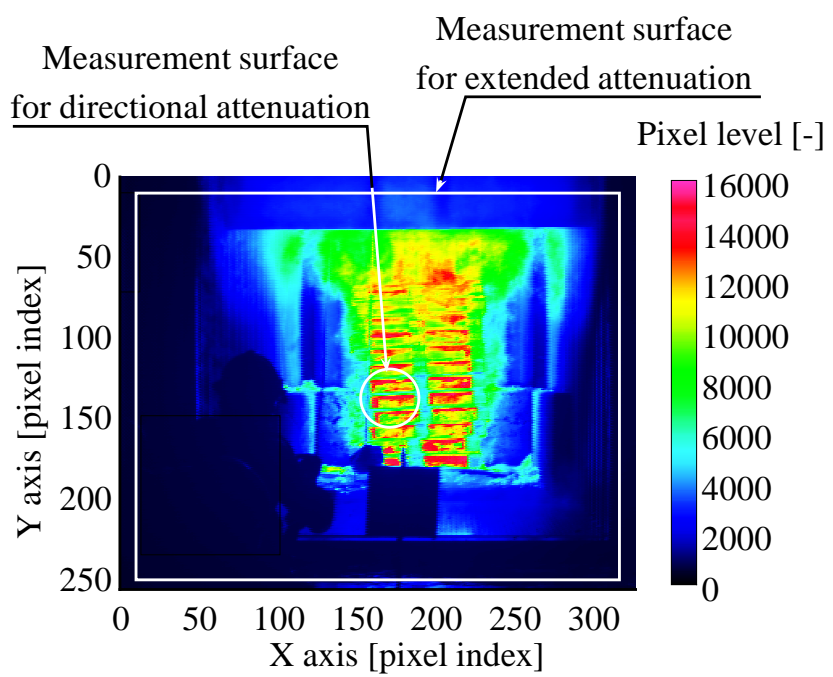

(a) Raw data from the IR camera for a "real" fire without spray.

Representation of the measurement areas for directional and extended attenuation estimations

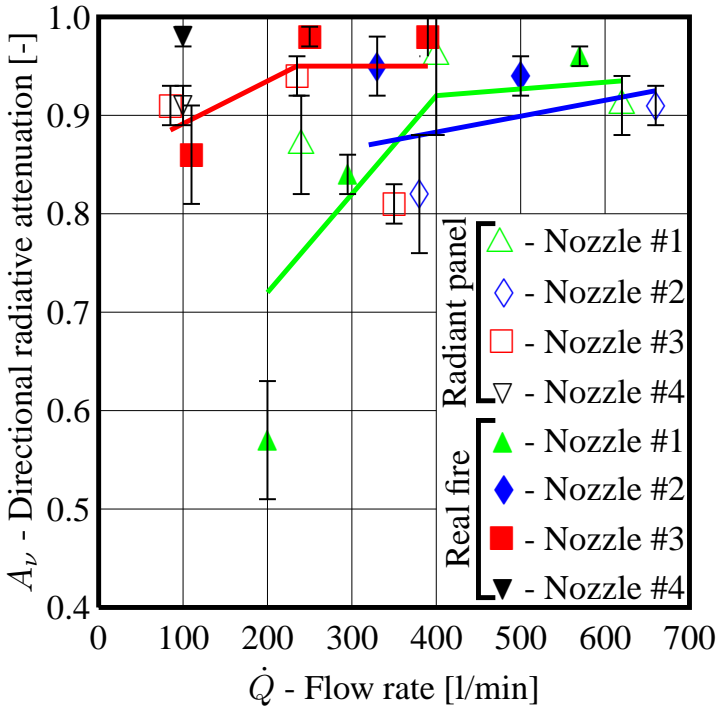

(b) Repeatability of the directional radiative attenuation estimation with a radiant panel and with a real fire measured at the wavenumber of $2564 \mathrm{~cm}^{-1}$ for each fire hose nozzle

Figure 8 Data and results provided by IR camera on a measurement surface equivalent to that for the spectrometer. $X$ and $Y$ axis denote the horizontal and vertical directions respectively

attenuation is estimated with the IR camera using a filter centred at $2564 \mathrm{~cm}^{-1}$. The results for a real fire are compared with the ones obtained for a radiant panel in Figure 8(b). Some discrepancies are seen but they are rather attributed 2 to variations with flow rates or the exact location of the experimental devices. However, the analysis shows the same 3 evolution of the radiative attenuation with the flow rate. Figure 8(b) shows that, even if the radiative source (real fire) 4 is not completely stable in time, the experimental protocol defined in this study allows to estimate directional radiative 5 attenuations correctly, considering that the data obtained with the stable radiant panel provides reference data.

6 The main disadvantage of the directional attenuation as calculated previously is that it overestimates the overall, effective, attenuation of the heat flux emitted by a large source of radiation. So the heat flux received by firefighters in such a 8 case, under $2 \pi$ sr, would be larger than the one measured under the normal incidence. To illustrate this comment, Figure 9 9(a) shows the directional radiative attenuation estimated for each pixel of the IR camera pictures. Here, to avoid post- 
processing pixels corresponding to positions where the radiation does not come from the fire, a threshold was applied on the pixel value, set at 1500. These pixels are in black in Figure 9(a) and are not concerned by the fire source. The directional attenuation in Figure 9(a) is then not homogeneous in space. The maximal value is observed at the centre of the spray (about 95\%) and the attenuation quickly decreases to reach values close to 50\%. Nevertheless, its representation seems to be asymmetric. Figure 9(b) proposes the directional radiative attenuations extracted from $\mathrm{X}$ and $\mathrm{Y}$ axes, as depicted by the white axes in Figure 9(a). These results demonstrate that the radiative attenuations decrease from 96\% (in the centre of the waterspray) to $52 \%$ (at the edge of the spray pattern). These results prove that the waterspray is clearly not homogeneous, due to local variations of the droplet volume fractions and/or the droplet size distributions. Moreover, the directional radiative attenuation is quite asymmetric, the highest values are observed according to $\mathrm{X}$ axis, due to the effects of the gravity on the spray features.

In the following, the IR camera was used to take into account the radiation coming from a large region (i.e., the entire front opening of the container, shown with a white rectangle in Figure 8(a)), which is equivalent to what the radiative sensor measures, placed behind the firefighters. This configuration is more realistic from the firefighter protection standpoint. In these conditions, the computed radiative attenuation is no longer directional but extended.

The results obtained based on the IR camera were compared with the ones obtained based on measurements with the radiative sensor (a thermopile). The latter provides a potential difference $(\Delta U)$ which is proportional to the net radiative heat flux impinging on the measuring area. A response coefficient $K$ allows converting the electric signal to the corresponding heat flux level. In addition, a joint K-thermocouple has been added to the sensor in order to estimate its temperature, named $T$, during the tests. Considering that the sensor behaves as a black surface, the total radiative heat flux is then estimated by:

$$
\varphi^{r}=\frac{\Delta U}{K}+\sigma T^{4}
$$

Knowing the radiative heat flux densities, the radiative attenuation can be computed using Eq. (6).

The performance of the sensors degrades over time and their response coefficient varies with the aging of the sensor as it is exposed to harsh environments. This is why, for each experimental campaign, these radiative sensors were re-calibrated 


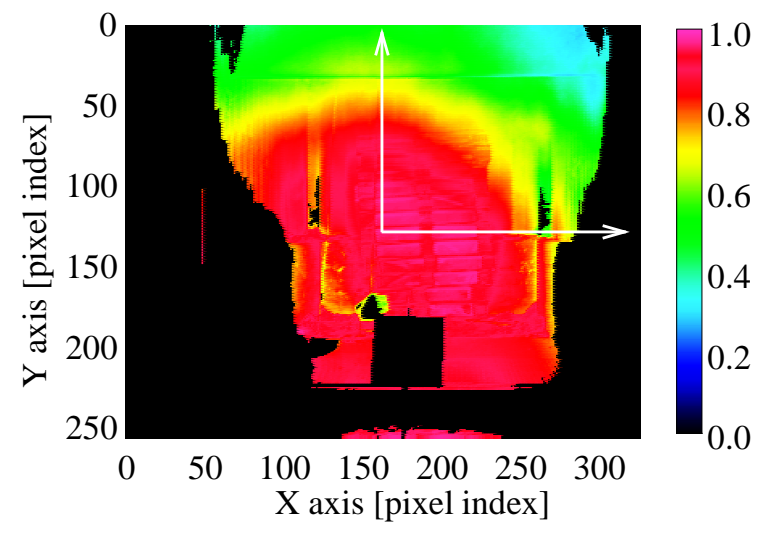

(a) Directional and spectral radiative attenuation for Nozzle \#3 obtained based on a measurement with the IR camera at the wavenumber of $2564 \mathrm{~cm}^{-1}$

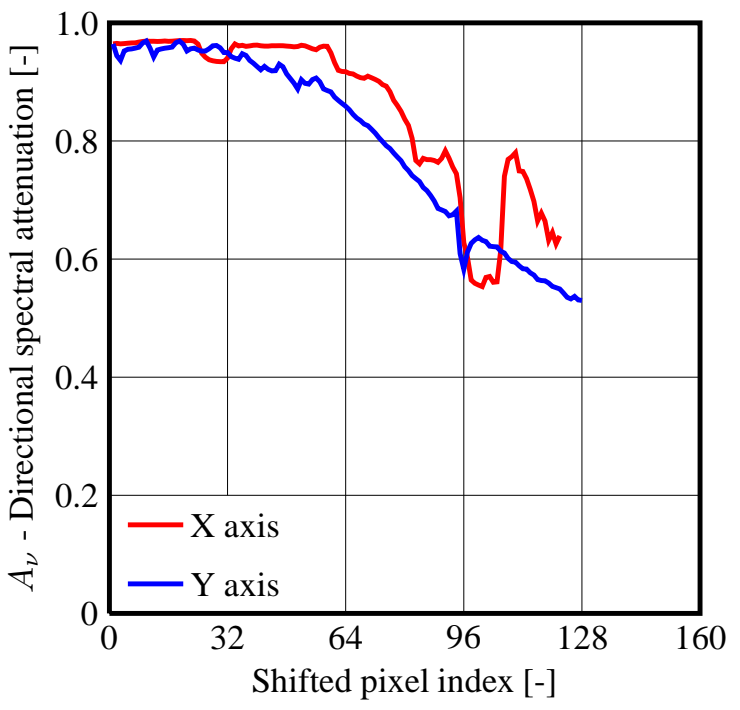

(b) Evolution of the directional and spectral radiative attenuation against the local position in $\mathrm{X}$ and $\mathrm{Y}$ directions

Figure 9 Evolution of the directional and spectral radiative attenuation inside the spray pattern for Nozzle \#3 at $\dot{Q}_{\text {int }} . X$ and $Y$ axis denote the horizontal and vertical directions respectively

with a dedicated experimental set-up, involving a calibrated black-body.

Figure 10(a) shows a comparison between the extended radiative attenuation obtained by the IR camera $\left(A_{\nu}\right)$ and the one estimated by a radiative sensor $(A)$. The spectral value of attenuation, set at a wavenumber of $2564 \mathrm{~cm}^{-1}$, can be compared with the total one due to the grey behaviour of radiative attenuation, previously showed. The maximal discrepancies between the experimental data (IR camera and radiative sensor) are here estimated at $14 \%$, suggesting that that a radiative sensor is a reliable experimental tool for evaluating the extended radiative attenuation in a simple manner.

These attenuation levels are lower than the ones estimated in the first part of this study (with up to $30 \%$ decrease in attenuation). This suggests that in the centre of the spray, the projected area provided by the droplets is larger (due to the higher number of droplets) than that at the edge of the spray, explaining the higher values of radiative attenuation at the centre of the spray. This also indicates that the smaller measurement area shown in Figs. 8(a) and 9(a) is not sufficient for quantifying the overall efficiency of the radiative shielding provided by the fire hose nozzles: the measurement area must 


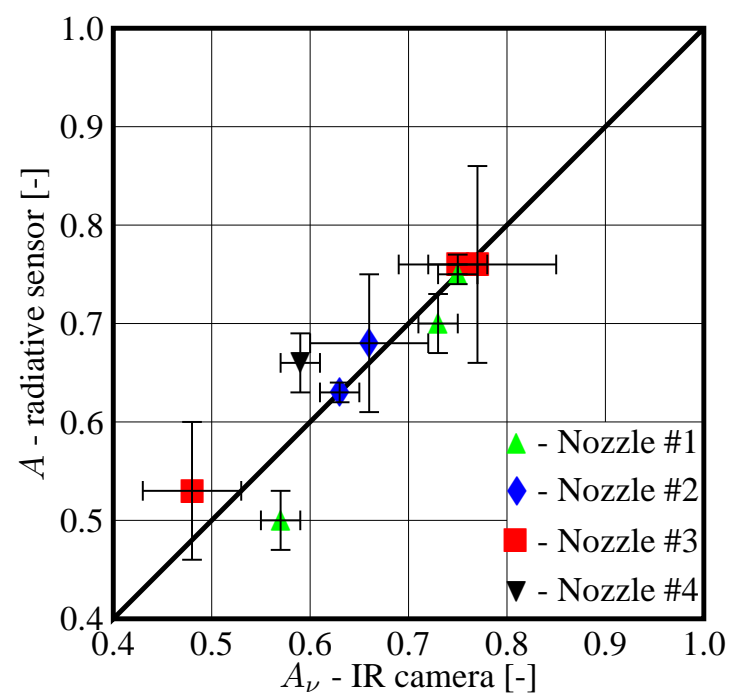

(a) Comparison of radiative attenuations based on IR camera $\left(A_{\nu}\right.$, at the wavenumber of $2564 \mathrm{~cm}^{-1}$ ) and radiative sensor $(A)$ measurements

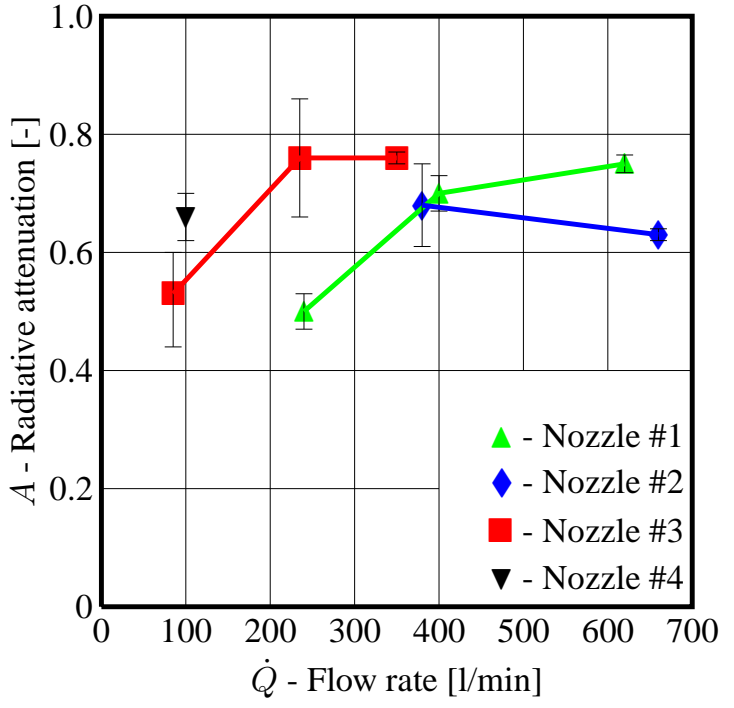

(b) Evolution of the radiative attenuation versus flow rate, based on radiative sensor measurements

Figure 10 Extended radiative attenuation estimation for all the sprays tested in the frame of this study

11 be extended to the whole fire region (see white rectangle shown in Fig. 9(a)).

1 Figure 10(b) shows how the total radiative attenuation varies against flow rate for each nozzle tested in this study. The

2 global trends are the same as the ones presented in Figure 8(b) with lower levels. The maximal radiative attenuation is

3 reached for Nozzle \#3 with 75\%. Globally, an increase in flow rate induces an increase in radiative attenuation, except for

4 Nozzle \#2, where a decrease of $5 \%$ attenuation is observed when the flow rate goes from $3801 / \mathrm{min}$ and $6601 / \mathrm{min}$. It is

5 surprising to see that the radiative attenuation of Nozzle \#3 is similar or even better than that of Nozzle \#1 for a flow rate

6 three to six times lower. It proves that increasing the total quantity of water is not the only parameter allowing to reach a

7 maximal attenuation. How the spray is generated and in particular how fine the droplets are have a major influence on the

8 obtained attenuation.

\section{CONCLUSIONS}

9 Radiative attenuation was evaluated for sprays generated by fire hose nozzles used by firefighters in operations. The aim

10 of this study was to define and test an experimental set-up to evaluate the radiative protection provided by these water 
sprays used as a radiative shield. At our best knowledge, this kind of studies at a full-size sprays for fire hose nozzles were never carried out.

For this study, four different fire hose nozzles were considered and selected from french rescue services, with flow rates between $80 \mathrm{l} / \mathrm{min}$ and $660 \mathrm{l} / \mathrm{min}$. The radiative attenuation was measured with an IR camera, a spectrometer and a total radiative sensor. Two types of radiative sources were used: a radiant panel and a "real" fire inside a standard shipping container

The results showed that the spectral attenuations are weakly decreasing with the wavenumber. For almost all the fire hose nozzles, increasing the flow rate induces an increase in the radiative attenuation.

7 The radiative attenuations estimated with the spectrometer, the IR camera and the radiative sensors demonstrate that the

radiative shielding is not uniform over the entire water spray region, suggesting that the droplet volume fraction and/or droplet size diameter change between the centre of the water spray and its edge. This observation justifies the use of an extended radiative attenuation rather than a directional one to provide relevant information on the thermal efficiency of these sprays as radiative shields for firefighters.

To estimate the radiative attenuation, the experimental set-up proposed by this study was the combination of a "real" fire inside a standard shipping container with a total radiative sensor (close to the fire hose nozzle) located $2 \mathrm{~m}$ in front of the container. In these experimental conditions, this study demonstrated that among all the nozzles tested, the maximal radiative attenuation is not greater than $75 \%$ for a fire hose nozzle at $2351 / \mathrm{min}$. This result can be used as a reference value to design new fire hose nozzles to improve their shielding efficiency against radiation.

The key parameters to promote a waterspray as an effective radiative shield are the mean droplet diameter (or the particle size distribution) and the droplet volume fraction. For the same quantity of water, the smaller droplets are more efficient for radiative attenuation than the bigger one. This observation is confirmed by the Mie theory for spherical particles [15]. Future work should focus on the characterization of these key parameters to better understand the different behaviour of the watersprays studied here.

On the other hand, future work should be dedicated to defining experimental set-ups in order to evaluate and quantify the capability of these sprays to cool hot smoke layers (to protect firefighters against the occurrence of flashover or fire gas 
ignition) and to estimate their efficiency for extinguishing fires from liquid or solid fuels.

\section{ACKNOWLEDGEMENTS}

1 The authors would like to thank Christophe Albert (BMPM), Christophe Alexandre (BMPM), Pierre-Louis Angeli 2 (BMPM), Jérémy Baillarge (SDIS 86), Frédéric Baridon (BMPM), Julien Bernadeau (SDIS 86), Sébastien Berteau 3 (BSPP), Vincent Bonvin (SDIS 54), Anthony Brexel (BSPP), Erwann Clouet (BSPP), Bruno Coudour (Institut P'), Cyrille 4 Deloy (SDIS 54), Stéphane Desroches (SDIS 86), Nicolas Dreuille (LCPP), Olivier Espejo (BSPP), Jean-Pierre Garo 5 (Institut P'), Maxime Gaud (SDIS 86), Franck Gaviot-Blanc (EFECTIS), Benoit Gellenoncourt (SDIS 54), Stéphane 6 Ghobrial (BSPP), David Guillo (BSPP), Erwan Hochet (SDIS 35), Kévin Huchet (SDIS 35), Antoine Maquart (BSPP), 7 Antoine Moutel (BSPP), Thierry Pascal (BMPM), Guillaume Pauza (BSPP), Alexandre Piel (SDIS 35), Alexandre Piquet 8 (SDIS 35), Nicolas Plazenet (BMPM), Steven Robert (SDIS 35), Olivier Roy (SDIS 35), Matthieu Tanneur (SDIS 86),

Julien Terrec (BSPP), Nicolas Trévisan (LEMTA), Julien Valdenaire (BSPP) and Anthony Vergne (BSPP) for their 10 valuable help when conducting tests. All members from LCPP and BSPP (Centre de formation du fort de la Briche) 11 are also thanked for providing technical and logistical support for these experimental campaign.

\section{REFERENCES}

[1] S. Särdqvist Water and other extinguishing agents Swedish Rescue Services Agency, Räddnings Verket, 2002.

[2] AFNOR - NF EN 15182-1+A1 Lances à main destinées aux services d'incendie et de secours Partie 1 : prescriptions communes, 25 p., 2010.

[3] AFNOR - NF EN 15182-2+A1 Lances à main destinées aux services d'incendie et de secours Partie 2 : lances mixtes à débit et jet réglables PN 16, 13 p., 2010.

[4] AFNOR - NF EN 15182-3+A1 Lances à main destinées aux services d'incendie et de secours Partie $3:$ lances à jet plein et/ou une diffusion à angle fixe PN 16, 10 p., 2010. 
[5] F. Vera, R. Rivera and C. Nunẽz Backward reaction force on a fire hose, myth or reality? Fire Technology, Vol. 51, pp. 1023-1027, 2015.

[6] F. Vera, R. Rivera and C. Nunẽz Backward Reaction Force in a Firehose Fire Technology, Vol. 54, pp. 811-818, 2018

[7] S.K. Chin, P.B. Sunderland and G. Jomaas Firefighter nozzle reaction. Fire Technology, Vol. 53, pp. 1907-1917, 2017.

[8] J. Sun, W. Li and M. He Analysis of fire water monitor jet reaction forces and their influences on the roll stabilities of urban firefighting vehicle Fire Technology, Vol. 55, pp. 2547-2566, 2019.

[9] S. Dembélé, J.X. Wen and J.F. Sacadura Experimental study of water sprays for the attenuation of fire thermal. ASME Journal of Heat Transfer, Vol. 123, pp. 534-543, 2001.

[10] A. Collin, P. Boulet, D. Lacroix and G. Jeandel On radiative transfer in water spray curtains using the discrete ordinates method. Journal of Quantitative Spectroscopy and Radiative Transfer, Vol. 92, pp. 85-110, 2005.

[11] A. Collin, P. Boulet, G. Parent and D. Lacroix Numerical simulation of a water spray. Radiation attenuation related to spray dynamics. International Journal of Thermal Sciences, Vol. 46, pp. 856-868, 2007.

[12] A. Collin, S. Lechêne, P. Boulet and G. Parent Water mist and radiation interactions: application to a water curtain used as a radiative shield Numerical Heat Transfer, Part A, Vol. 57, pp. 537-553, 2010.

[13] C.C. Tseng and R. Viskanta Absorptance and transmittance of water spray/mist curtains Fire Safety Journal, Vol. 42, pp. 106-114, 2007.

[14] R. Mehaddi and A. Collin and P. Boulet and Z. Acem and J. Telassamou and S. Becker and F. Demeurie and J.-Y. Morel Use of a water mist for smoke confinement and radiation shielding in case of fire during tunnel construction International Journal of Thermal Sciences, Vol. 148, 106156, 2020. 
[15] C.F. Bohren and D.R. Huffman Absorption and scattering of light by small particles New York : John Willey and Sons, 1983.

2 [16] M.F. Modest Radiative Heat Transfer 2nd edition, Academic Press, New York, NY, USA, 2003.

3 [17] G. Parent, P. Boulet, R. Morlon and E. Blanchard Radiation attenuation and opacity in smoke and water sprays $J$. $4 \quad$ Quant. Spectrosc. Radiat. Transfer, Vol. 197, pp. 60-67, 2017.

5 [18] G. Parent, P. Boulet, S. Gauthier, J. Blaise and A. Collin Experimental investigation of radiation transmission through a water spray Journal of Quantitative Spectroscopy and Radiative Transfer, Vol. 97, pp. 126-141, 2006. 